\title{
STEM Optical Sectioning for Imaging Screw Displacements in Dislocation Core Structures
}

\author{
Peter D Nellist ${ }^{1,2}$, Hao Yang ${ }^{1}$, Juan G Lozano ${ }^{1}$, Timothy J Pennycook ${ }^{12}$ and Peter B Hirsch ${ }^{1}$ \\ 1. University of Oxford, Department of Materials, Oxford, UK. \\ 2. EPSRC SuperSTEM Facility, Daresbury Laboratory, Warrington, WA4 4AD.
}

Edge dislocations are characterised by columns of atoms in tension or in compression in a plane normal to the dislocation, above and below the slip plane. Such dislocations are conventionally viewed end-on at high resolution in transmission electron microscopes, because the tensile and compressive displacements are clearly visible. Screw dislocations are characterised by equivalent atoms in the columns parallel to the screw dislocations being displaced along the screw axis to form a helix. They cannot be observed in end-on images because the displacements are parallel to the viewing direction. In this paper we show that the helical displacements around a screw can be imaged with the dislocation lying in the plane of the transverse to the electron beam by optical sectioning in high-angle annular dark-field (HAADF) scanning transmission electron microscope (STEM) imaging. This novel technique is illustrated by application to the measurement of the c-component in the dissociation reaction of a mixed [c+a] dislocation in GaN that has previously been observed end-on [1].

The development of correctors for the spherical aberration in STEM has led to a dramatic improvement in spatial resolution (see for example [2]), but alongside comes an even greater reduction in the microscope depth of focus, which in a state of the art instrument may just be a few nanometres, and less than the thickness of the sample. An opportunity is therefore created to focus on specific depths within the sample and to extract information from just that layer - a process referred to as optical sectioning. Achieving the full depth resolution available for a general sample requires a confocal configuration, but at atomic resolution a conventional STEM configuration can be used [3].

Figure 1 shows layers extracted from a model of c-type (screw) dislocation aligned along the c axis [0001] in GaN. Each layer consists of an extracted ( $2 \overline{1} \overline{1} 0)$ plane (also referred to as the a plane), and are therefore parallel to the dislocation line. In layers far from the dislocation, the screw displacements vary slowly across the field of view as expected from the lower shear strain that exists further from the dislocation core. Layers close to the dislocation core show screw displacements that very rapidly in the vicinity of the dislocation core, with a rapid shearing of the (0002) planes to given an apparent displacement of $\mathbf{c} / 2$ across the dislocation core (as expected for a total Burgers vector of c).

A focal series of experimental images were recorded using a Nion UltraSTEM100 aberration-corrected STEM operating at $100 \mathrm{kV}$ (Figure 2). A $1 \mu \mathrm{m}$ thick sample of GaN, grown by metalorganic vapour phase epitaxy on a sapphire substrate, was thinned to be viewed along the a crystallographic axis. A dislocation was found lying in the plane of the sample, and characterized using weak-beam imaging to be of a mixed (c+a) type. As the electron beam is focused closer to the dislocation from (a) to (e), the shearing of the (0002) planes becomes more localized in the image, and a more detailed observation of the screw displacements following Fourier filtering shows that the shearing occurs along two distinct lines lying perpendicular to the (0002) planes. It is therefore apparent that the dislocation core has dissociated, and measurement of the displacements allows the full dissociation reaction to be deduced $[4,5]$. 
[1] P.B. Hirsch et al., The dissociation of the [a+c] dislocation in GaN, Philosophical Magazine, 93 (2013) 3925.

[2] P.D. Nellist et al., Direct Sub-Angstrom Imaging of a Crystal Lattice, Science, 305 (2004) 1741.

[3] P.D. Nellist and P. Wang, Optical Sectioning and Confocal Imaging and Analysis in the

Transmission Electron Microscope Annual Review of Materials Research, 42 (2012) 125.

[4] H. Yang et al., manuscript in preparation.

[5] The authors would like to acknowledge financial support from the EPSRC (grant number

EP/K032518/1) and the EU Seventh Framework Programme: ESTEEM2.

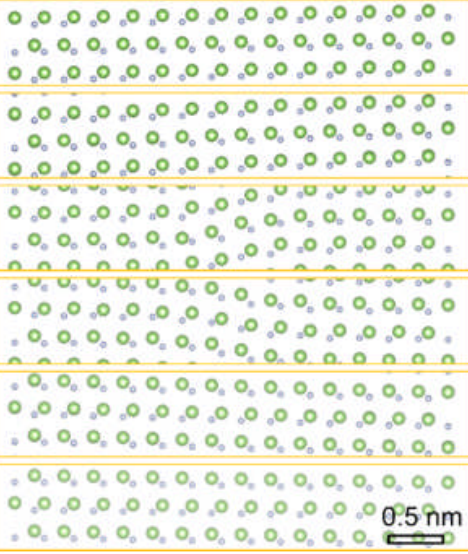

Figure 2. Optical sectioning STEM ADF images of a [c+a] dissociated screw dislocation viewed perpendicular to the dislocation line along the a direction. The focal step in (a) (c) and (e) is $4 \mathrm{~nm}$ each. (b)(d) and (f) contain Fourier filtered versions of the corresponding images using just the (0002) Fourier components to highlight the shearing of the planes.
Figure 1. Structure model of a $10 \mathrm{~nm}$ thick GaN c type screw dislocation viewed at different depths along [2 $\overline{1} \overline{1} 0]$ direction, with the screw dislocation lying in the middle. From the top to the bottom panel, the distances from the dislocation core are 5, 1, 0.3, 0.3, 1 and 5nm, respectively.
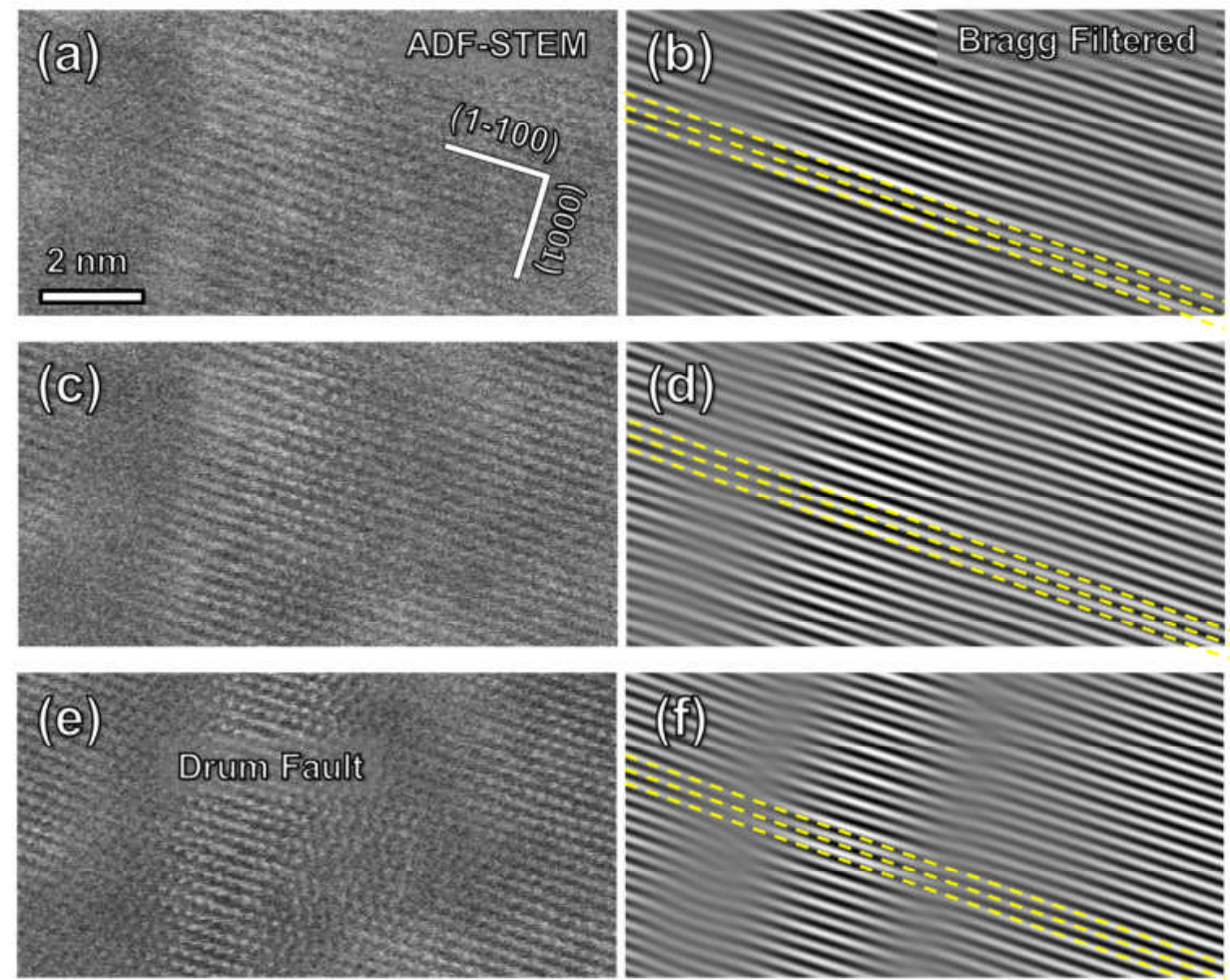